\title{
Language and Speech in Tourette Syndrome: Phenotype and Phenomenology
}

\author{
Larry Burd
}

Published online: 27 August 2014

(C) Springer International Publishing Switzerland 2014

\begin{abstract}
In this update we review our past publications on this topic and add new information on Tourette syndrome (TS) and speech and language disorders. The phenotype for TS is comprised of vocal and motor tics. The vocal tics are expressed as simple and complex, and in nearly all cases share the same neurolinguistic mechanisms of speech and language for insertion into speech and discourse. The tics are typically inserted into ongoing speech at pauses, points of change in inflection, and are typically without meaning. Adherence to segmental, lexical, and suprasegmental rules of linguistics suggest the role of tic selection; expression and elimination of tics involves multiple linguistic mechanisms which are poorly understood. TS increases the risk for clinically significant speech and language disorders.
\end{abstract}

Keywords Vocal tics $\cdot$ Coprophenomena $\cdot$ Tic selection · Dysfluencies · Phonology · Pragmatics · Expressive language $\cdot$ Learning disabilities

\section{Introduction}

Tourette syndrome (TS) is a neuropsychiatric developmental disorder with onset in childhood, with the first case-series being described in 1885 [1]. The disorder is manifest as vocal and motor tics of highly variable severity which do not have to present concurrently [2]. In Table 1 we summarize the phenomenological features of vocal tics. In general, the nosological characteristics of simple tics are of brief duration and are comprised of a single action or vocalization, while complex

\section{Burd $(\bowtie)$}

Department of Pediatrics, University of North Dakota School of Medicine and Health Sciences, 501 N Columbia Road, Stop 9037, Grand Forks, ND 58202-9037, USA

e-mail: larry.burd@med.und.edu tics are often longer in duration and are comprised of multiple actions or vocalizations, which are sequenced in a highly stereotyped presentation. While tic expression can consist of an impressive range of movements and vocalizations, the most common tics observed in TS are summarized in Table 2.

In most cases, the developmental trajectory of TS is one of tic onset at around 5 years of age which is remarkably similar across gender, countries, and clinics [3]. A peak in symptom severity typically occurs by $12-13$ years of age ( $80 \%$ of people), with a gradual decline in symptom severity in late childhood and continuing through adolescence [4-6].

The epidemiological characteristics of TS have led to important advances in our understanding of associated disorders and impairments which are generally conceptualized as cooccurring conditions (comorbidity) [6-9]. While TS has attracted a number of researchers in genetics, epidemiology, neurology, neuroanatomy, and clinical management, one area that has received much less attention has been the neuropsycholinguistic features of tics and TS [10, 11]. In this chapter, I will update our previous publications on this topic and discuss the syndromic features of TS that involve speech and language $[10,11]$. The chapter is organized around the psycholinguistic components of TS.

\section{Tic Selection and Expression}

In clinical practice with children who have TS and other tics, parents and teachers often ask 'why does he do that?' The simplistic attribution of this behavior to 'it's a tic' is unsatisfactory for them and us and offers surprisingly little useful information on differentiation of behavior problems from developmental disorders. We have remarkably little information on how tics arise, how they are selected for expression, or what factors are involved in the attenuation of their expression. Figure 1 is a conceptual model of pathways for tic selection 
Table 1 Phenomenological variables of the characteristic features of tics ${ }^{\mathrm{a}}$

Range from voluntary to involuntary

Often interruptive

Repetitive but often occurs in clusters

Can be inhibited

Can be induced

Worsened by stress

Emotionally loaded content

Waxing and waning

In adults, more chronic tics (less waxing and waning)

Purposelessness to purposeful (discharging sensory phenomena)

Can be situational in expression

${ }^{\text {a }}$ Not all are required and some characteristics may apply in only some situations

and expression. While this model encompasses the role of multiple-effect modifiers, it still seems inadequate when one tries to understand the role of tics and TS on severity of multiple neuropsychiatric phenotypes. Severity and the developmental course of these disorders are highly variable in clinical practice.

\section{Insertion and Incorporation of Vocal Ties into Speech}

Vocal and motor tics are rarely interruptive of words or phrases and are often inserted into ongoing speech at points of emphasis, including pauses for punctuation, between large units of speech, or at points of modulation of speech. Thus, the suprasegemental elements of speech are utilized during insertion of vocal tics into speech. Most complex vocal tics consist of inserted or incorporated phrases that adhere to phonological and syntactic rules of speech formulation. However, both simple and complex tics often appear to violate semantic and pragmatic rules since they typically convey no obvious meaning in the context of the larger units of conversation where they occur. Indeed this stereotyped expression, lack of meaning, and lack of derailment of ongoing speech are key nosological factors in tic detection and classification of vocal tics and are especially important for the identification of complex tics consisting of phrases.

\section{Speech Abnormalities}

Abwender et al. reported a history of tics in 11 of 22 subjects $(50 \%)$ with dysfluency [12]. They noted that tics and dysfluencies are both inherited, are more common in males, have a childhood onset, have a developmental course with improvement over time, have a waxing and waning course, can be partially suppressed, and both tend to be exacerbated
Table 2 Common vocal tics in Tourette syndrome

\begin{tabular}{ll}
\hline Simple tics & Complex tics \\
\hline Vocal tics & \\
Throat clearing & Repeating parts of words, or phrases \\
Sniffing & Animal sounds \\
Spitting & Prosodic changes \\
Coughing & Stuttering \\
Yelling & Talking to oneself with multiple \\
& characters assuming \\
& different intonations \\
Hiccupping & Derogatory phrases or statements \\
Snorting & \\
Animal sounds (e.g. barking) & \\
Belching & \\
Difficult-to-classify behaviors & \\
Finger snapping & \\
Clapping & \\
Rhythmic tapping & \\
Mental sounds & \\
Hearing repetitive words & \\
Repetitive sounds & \\
Silent palilalia & \\
\hline
\end{tabular}

by stress [12]. A case report of tics and dysfluency was also reported by Van Borsel and Vanryckeghem [18]. Previous research has identified an increased prevalence of selfreported dysfluencies in patients with TS [13•]. Meige and Feindel have also suggested a complex relationship between

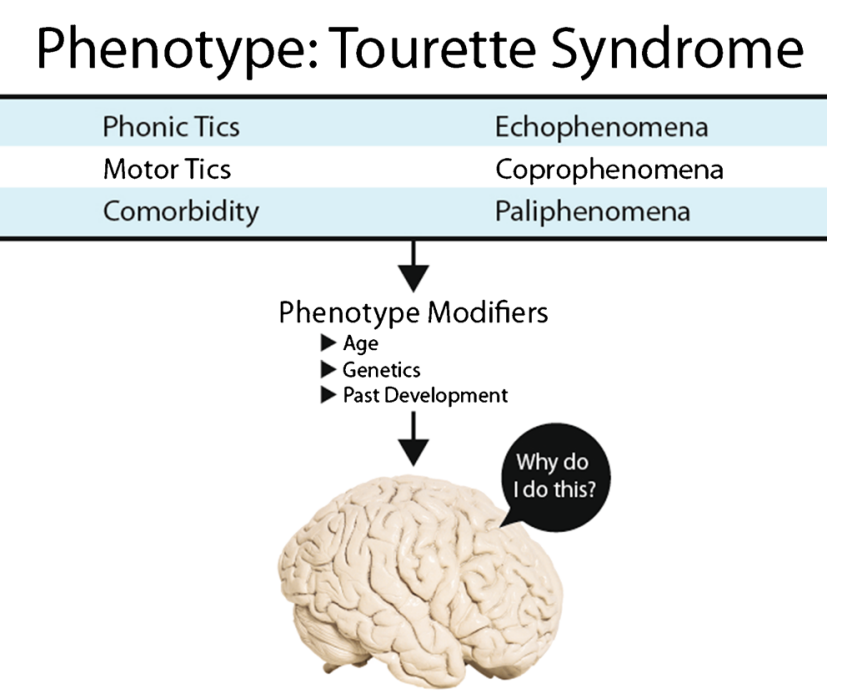

Fig. 1 A theoretical model of the variables and interactions of phenotype expression in TS. These factors interact and are modified by past development, age, severity, and comorbidity. The factors regulating tic selection and patterns of expression in TS and other tic disorders are not yet completely understood. TS Tourette syndrome 
TS and dysfluency [14••]. They also identified prosodic abnormalities, and intrusive mental tics consisting of words, phrases, and sounds in TS and other tic disorders [14*0]. A case report of an 18-year-old college student reported that he had improvement in his dysfluency but not his tics when he pierced his tongue and inserted a tongue stud. He noted that when the stud was removed the dysfluency returned. This relationship was repeated several times [15].

The prevalence of dysfluency in TS ranges from $7.7 \%$ of subjects with TS in the Tourette International Consortium (TIC) data to $15-31 \%$ in other studies [3, 9, 16, 17]. Logistic modeling of tic severity resulted in a best-fit model for severe tics which included age of onset, age at diagnosis, gender, perinatal problem, and the specific comorbidities of obsessive-compulsive disorder, mood disorders, anxiety disorders, coprolalia, copropraxia, stuttering, and sexually inappropriate behavior [16]. When the logistic modeling for tic severity was constrained to the 21 neuropsychiatric disorders in the TIC database, the model was comprised of obsessivecompulsive behaviors, mood disorders, anxiety, coprophenomena, stuttering, sexually inappropriate behavior, and intellectual disability [16]. This suggests that the presence of dysfluency in TS appears to be a useful marker for risk of increased syndromal severity and functional impairment.

While dysfluency may, in some cases, be a manifestation of the spectrum of tic disorders, we still do not know how tics and dysfluency are related. Does dysfluency in the context of tic disorders have a different neurobiological or genetic basis or are some tics atypical dysfluencies [10, 11]? Alternatively, other researchers have suggested that the dysfluency seen in TS is not characteristic of developmental stuttering. They suggested that the behaviors, hesitations, false starts, interjections, and phrase repetitions are not characteristic of the syndrome of stuttering when current strict criteria for stuttering are applied [18].

Another speech abnormality we have examined is the relationship between abnormalities in prosody and voice abnormalities (mutational falsetto) and tics [19]. It is interesting that both of these patients also presented with moderate to severe echolalia. Both had a positive response to pharmacological intervention and psychoeducational therapy targeted at their individual developmental needs.

\section{Expressive and Receptive Language Impairments}

Ludlow et al. reported deficits in language expression and comprehension in subjects with TS compared with matched controls [20]. The potential complexity of the relationship between tics and language development was also demonstrated in a study by Fisher and colleagues [21]. In this study, four patients with a distinctive syndromal disorder comprised of (1) pervasive developmental disorder; (2) tic disorders, including TS; (3) a characteristic pattern of speech and language impairment, including severe delays in both receptive and expressive language; (4) mumbling; and (5) finger flicking were treated with the dopamine blocker haloperidol. All four patients demonstrated broad improvement with large gains in acquisition of speech and improvement in both receptive and expressive language [21]. Discontinuation of the medication in one patient resulted in regression of the previous gains from treatment; however, restarting the medication reproduced the previous improvement in his speech and language.

Another interesting case report describes an intralaryngeal injection of botulinum toxin which resulted in improvement in coprolalia [22]. The pathophysiology of this intervention is not readily apparent. Alternatively, some patients have the onset of tics after exposure to some medications [23, 24].

\section{Coprophenomena}

The coprophenomena include coprolalia and copropraxia and, in some patients, reports of ideation of stereotyped sexual gestures, thoughts, and actions. Coprolalia primarily consists of scatologic words and racial or social epithets [25]. Coprolalia occurred at some point in $20.6 \%$ of males and $14.9 \%$ of females; copropraxia in $7.2 \%$ of males and $4.6 \%$ of females in the TIC data [26 $6^{\circ}$. The mean onset of coprophenomena was about 5 years after the onset of tics, and in $11 \%$ of those with coprolalia and $13 \%$ of those with copropraxia this was the initial symptom of TS [3]. The inclusion of words and phrase content for the expression of coprolalia appear to be dependent on regional cultural knowledge of the same [29]. One study provided data on coprolalia from 11 different nationalities and found that the coprolalic words or phrases differed by country. Shapiro also noted that patients with TS tend to utilize the most socially unacceptable terms within their own cultural context [27]. Thus, coprolalia seems to be defined by the appreciation and utilization of a sophisticated region- and culture-specific lexicology of meaning and inferred intentionality of speech, phrases and, in some cases, gestures. Jankovic and Fahn reported on a patient who had coprolalia in Spanish at home around her family and coprolalia in English when she was in the hospital [28]. We have cared for a patient with severe mental retardation who was nearly non-verbal but had coprolalia in German (his first language and the primary language of his family). Nearly half of the 15-20 words he was capable of saying were mostly coprolalic in German. Case reports of tics and coprolalia emerging after treatment with neuroleptics has been reported [29]. The same has also been noted for copropraxia.

Van Lancker and Cummings have suggested that coprolalia in TS be considered as ego alien when the word or phrase is unwanted and intrusive [29]. 
In our clinical practice we have observed an updating of the corpus of both copropraxia and coprolalia over time which appears to be a response to changes in societal definitions of behaviors which are rapidly acquired and utilized by patients. The cultural, situational, and social context of coprophenomena suggests that we have much to learn about how tics are selected for expression and the broad array of factors which influence the use of some specific tics. The thought that these are simply aberrant motor programs seems to be at odds with what we now appreciate about coprophenomena. Complex developmental factors regulate tic selection and emergence which are influenced by an array of inter- and intrapersonal complexities of social norms and changes in societal linguistic attributions.

\section{Writing and Tics}

In 1907, Meige and Feindel reported that some patients found that writing could induce the use of specific vocal tics [14*0]. Ludlow et al. reported deficits in written language expression in subjects with TS compared with ageand sex-matched controls [20]. De Nil et al. [13•] found that children with TS self-reported difficulty in written language expression. Lang et al. reported one patient with TS who had tics that were expressed by typing [30]. Some people with TS demonstrate coprographia $[14 \bullet \bullet, 31]$. In our experience, this may include repetitive drawing of figures or events, some of which may be copropraxic in content.

\section{Signing and Tics}

Several reports have identified signed tics in people with deafness. Lang et al. [30] reported on a patient who developed tics at 8 years of age, and by 17 years of age she began training to learn sign language, and then began to incorporate tics into her use of sign language. She later developed coprolalia and copropraxia in her signing. This would also occur when she was not talking or thinking about the word or phrase she was signing, and analogously to persons using speech, some of her coprolalic signs after much usage were at times truncated.

One report of a 31-year-old man with prelingual deafness who had motor and vocal tics also had coprolalia, expressed through his sign language. He felt embarrassed about the compulsion and often tried to disguise it as another sign. Rickards noted that the urge to swear and attempts to disguise it in the context of other behaviors are common in both signing and spoken language [32].
Another report was of a patient who was found to be profoundly deaf at the age of 10 months [33]. At 5 years of age he was first noted to make screeching noises, and repetitively making 'ppp' or 'prrrr' sounds. He had multiple facial twitches. At 7 years of age he was taught sign language and finger spelling, and psychometric testing found him to have an IQ of 120 . At the 20 years of age his tic behavior markedly worsened and his parents felt that he had developed TS. He made frequent loud screeching noises and complex motor movements. It became clear that sign language was an integral part of the expression of his range of tics. He would randomly intersperse obscene signs and finger-spelled obscene words in normal conversation. $\mathrm{He}$ repeatedly produced sexual signs when conversing with women. The production of contextually sensitive tics and finger-spelled obscenities was felt to be equivalent to writing or spelling words out. In this context, the semantic features are divorced from phonologic content. This suggests that the conceptual ideas underlying coprolalia may be more important than the phonology of the utterance.

Another report was of a patient who at the age of 4 months was diagnosed as profoundly deaf [34]. He neverr developed any vocal language. At 7 years of age he was referred for simple motor tics (eye blinking) and simple vocal tics (sniffing and throat clearing). He developed copropraxia, consisting of obscene gestures which were not sign language. His parents noticed 'coprolalia' in his sign language, palilalia, repeating his own signs, and 'echolalia', repeating signs made by others. Since he has never had a vocal language due to congenital profound deafness, the fact that he developed obscene expressions in sign language might best be captured by the term, coprolaliopraxia.

A 23-year-old woman first developed motor tics when she was 8 years of age [30]. Vocal tics began at 12 years of age with low-volume sounds, and at 17 years of age she developed yelping vocalizations. She began formal training in sign language at 17 years of age, and by the age of 18 years she began signing obscenities. Usually the complete sign of 'fuck' or 'shit' was combined with a high-pitched vocalization of shortened forms of the obscenities (e.g. 'fu' and 'sh'). Only later, after she had been signing obscenities for a time, did she develop more typical copropraxia; for example, lifting the middle finger. Given her use of motor actions (praxis) in the performance of the verbal symptoms of coprolalia, echolalia, and palilalia, Lang et al. suggested the terms 'coprolaliopraxia', 'echolaliopraxia', and 'palilaliopraxia' to denote these unusual, complex motor-vocal tics.

Dr. Roger Freeman, who has 40 years of experience with deaf persons, notes that fingerspelling, if it involves poorly controlled, unacceptable written words 'in the air', is a form of coprographia, not coprolalia; whereas signing (gestural verbal language) would be coprolalia. Signing is verbal, and deaf 
persons (native signers) also use gestures, like anyone else, that are not verbal-gestural 'words' or verbal; they could thus have copropraxia in their gestural communication. When signs for concepts or proper nouns are not in a deaf person's vocabulary, they fingerspell it (or in a hearing person's vocabulary when it is limited, when they are learning to sign) [Freeman R, personal communication, 2014].

\section{Situation-Specific Tics}

Some tics appear to be situation-specific. We have encountered several patients who have a tic repertoire used only in specific situations. This may involve a person uttering phrases specific to being around or thinking about a person in situations where specific tics are uttered or expressed. Some patients report the development of long-lasting tics initiated by exposure to a specific person or situation. For some people this may be a particular behavior, personal characteristic, or physical feature of a person. For example, the author has had multiple children comment on his lack of hair ('shiny head') and his 'chubby cheeks'. In some cases these comments are blurted out followed by an apology, only to be repeated over and over again. Children note that this can be a serious problem in the presence of authority figures who are unable to appreciate the difficulty children with TS can have in modulating these utterances.

el-Assra reported on a 13-year-old whose tics apparently began after exposure to a traumatic incident [35]. Many children and adults have reported that they have 'picked up' tics from a person or situation. These tics may include prosodic mannerisms, a mispronounced word, or a behavior which may be imitated for years and, in some situations, only occurs in the presence of an individual or situation. Among children with TS who have behaviors that persist in the presence of significant consequences, it may be useful to review this behavioral manifestation of TS.

\section{Learning Disabilities}

Increased prevalence rates of learning disabilities (LDs) have been reported in people with TS $[9,19,20,36,37]$. In a recent cohort study of TS, the prevalence of LDs was $51 \%$ [38]. In this cohort, $21 \%$ met the criteria for two or more LDs, and $11 \%$ had three or more LDs [38]. The rate of severe LDs (defined by a 2 -standard-deviation discrepancy between IQ and academic achievement) was $21 \%$ for one $\mathrm{LD}$, and $9.5 \%$ for two or more LDs, and nearly $3 \%$ of children met the criteria for three LDs.

The North Dakota cohort studies were then confirmed by the TIC database where the rate of LDs was $22.7 \%$, which is nearly five times the expected rate [38]. In this study, LDs and
TS were associated with a mean of three additional comorbid disorders (attention-deficit hyperactivity disorder [ADHD], anger, sleep, mood, social skill impairments, anxiety, increased rates of inappropriate sexual behavior, conduct disorder, coprolalia, pervasive developmental disorders, and psychosis) [16]. TS can be expressed as a broad phenotype comprised of increased comorbidity. The broad phenotype expression of TS is supported by increases in prevalence rates of comorbid disorders when compared with the TS-only population (ADHD 80.2\%, compared with $51 \%$ in the TS and no LD group, anger problems $46.3 \%$ vs. $33.8 \%$, and sleep disturbance $30.2 \%$ vs. $23.7 \%$ in the TS and no LD group) [16]. A logistic regression model from this study identified a five-variable model for TS and LD: (1) being seen for evaluation before 18 years of age; (2) male gender; (3) decreased proportion of family members with tics or TS; (4) increased rates of perinatal adversity; and (5) increased rate of total comorbid disorders. De Nil et al. [11] reported that children with TS indicate they have difficulty with a wide range of educationally-required skills. This was an interesting methodology since the authors asked children to self-report their difficulties. This study found increased rates of difficulty in speech and language formulation in expressive, receptive, and written language. It is interesting to note that the children also reported problems in word finding, oral expression, reading, stuttering, and in saying certain letters.

In children with TS, the severity of learning impairments is high and the prevalence of severe LDs is increased between 5 and 15 times the expected rates. This model suggests a genetic/environmental interaction for the expressed phenotype of tics/TS and LDs.

\section{Conclusions}

TS and speech, language, and communicational disorders are common disorders with highly variable severity. While the precise nature of the relationship between the two is unclear, it is clear that TS increases the risk for clinically significant speech and language disorders. This comorbidity appears to encompass interactions among neurocognitive, affective, and neurobiological relationships. These interactions may be useful in understanding the pathophysiology across development for neuropsychiatric disorders in general. In TS, the relationship between speech, language, and vocal tics (including coprolalia) seems to be an unusually compelling area for further study of the quiddity of self. Further research in this area may also be very useful in advancing our knowledge in the area of how mind-brain transduction functions in the development of speech, language, and intentionality. How do the biological and social architectures of systems and relationships change when they are perturbed? Thus, how do tics come about? Are the tics aberrant neurocognitive 
impulses that are poorly regulated and inadequately inhibited, or are tics selected for expression? Either model has definite weaknesses.

TS is a dynamic disorder where the phenotype interacts with past development and experience in many cases. Several important concepts require additional thought and emphasis from researchers and clinicians.

1. What are the phenomenological and phonological boundaries of a vocal tic? Do finger snapping, sniffing, belching, flatus, or silent palilalia qualify as vocal or motor tics? Must vocal tics be accompanied by a sound? What about the patient who has tics and hears a mental sound repeated over and over and reports that it is 'just like a tic'? If palilalia is a vocal tic, how are we to classify silent or mental palilalia?

2. Since the early descriptions of tics, the concept of 'mental tics' has repeatedly been mentioned [14••]. Do tics require action movement or sound to be classified as a tic? If the response is 'yes' what are we to call these mental phenomena that patients describe as 'just like a tic only in my head', and if one answers 'no', how might one conceptualize a nosology of the mental experiences that would meet criteria for a mental tic? How are we to classify repetitive thoughts about words, sounds, or phrases? The future study of tics to determine if they can occur without observable expression seems to parallel our current difficulties in understanding thought disorders in young children, in non-verbal people, or in people with severe cognitive impairment.

3. Increased emphasis on how tics originate and how tics are selected for expression are needed. How does exposure to tic-provoking factors (stress, anxiety, or social situations) modify tic expression? Why do some words, sounds, and phrases frequently manifest as tics and not others? Do tics pass clinical thresholds, as can obsessions or compulsions, and why are some commonly forbidden utterances or actions expressed as tics, but not others? Why are some (vocalizations or actions) externalized and others constrained to expression within (mental tics)? Why do some tics start after exposure to a situation, event, or person? Why are some tics only seen in these certain situations? Why do some specific tics have a familial expression across generations?

Clearly, we have much to learn about the relationship between the effects of past development, TS and speech, language, and communication disorders as effect modifiers for the individual TS phenotype. The advent of new therapeutic approaches, especially the habit reversal therapies, will also advance our understanding about tics and related disorders $[39 \bullet \bullet, 40]$. TS provides a unique and compelling clinical entity to develop parallel and complementary studies on the confluence of genetic factors and environmental influences in both neurodevelopmental disorders and mental illness.

\section{Compliance with Ethics Guidelines}

Conflict of Interest Larry Burd declares that he has no conflict of interest.

Human and Animal Rights and Informed Consent This article does not contain any studies with human or animal subjects performed by any of the authors.

\section{References}

Papers of particular interest, published recently, have been highlighted as:

- Of importance

•. Of major importance

1. de la Tourette G. Étude sur une affection nerveuse caracterisée par de l'incoordination motrice acommpagnée d'echolalie. Arch Neurol. 1885;9:19-42. 153-156.

2. American Psychiatric Association. Diagnostic and statistical manual of mental disorders. 5th ed. Washington, DC: American Psychiatric Association; 2013.

3. Freeman RD, Fast DK, Burd L, Kerbeshian J, Robertson MM, Sandor P. An international perspective on Tourette syndrome: selected findings from 3,500 individuals in 22 countries. Dev Med Child Neurol. 2000;42(7):436-47. The first very large pooled data set for TS.

4. Burd L, Kerbeshian J, Barth A, Klug MG, Avery PK, Benz B. Long-term follow-up of an epidemiologically defined cohort of patients with Tourette syndrome. J Child Neurol. 2001;16(6):4317.

5. Leckman JF, Zhang H, Vitale A, Lahnin F, Lynch K, Bondi C, et al. Course of tic severity in Tourette syndrome: the first two decades. Pediatrics. 1998;102(1):14-9.

6. Kerbeshian J, Burd L. Tourette syndrome: a developmental psychobiologic view. J Dev Phys Disabil. 1994;6:203-18.

7. Martino D, Madhusudan N, Zis P, Cavanna AE. An introduction to the clinical phenomenology of Tourette syndrome. Int Rev Neurobiol. 2013;112:1-33.

8. Cohen DJ, Leckman JF, Pauls D. Neuropsychiatric disorders of childhood: Tourette's syndrome as a model. Acta Paediatr Suppl. 1997;422(0803-5326):106-11.

9. Comings DE, Comings BG. TS, learning, and speech problems. J Am Acad Child Adolesc Psychiatry. 1994;33(3):429-30.

10. Burd L, Leech C, Kerbeshian J, Gascon GG. A review of the relationship between Gilles de la Tourette Syndrome and speech and language. J Dev Phys Disabil. 1994;6(3):1-19.

11. Burd L, Christensen T, Kerbeshian J. Speech, language, and communication in Tourette's Syndrome. Annu Rev Appl Linguist. 2008;28(1):170-90.

12. Abwender DA, Trinidad KS, Jones KR, Como PG, Hymes E, Kurlan R. Features resembling Tourette's syndrome in developmental stutterers. Brain Lang. 1998;62(3):455-64.

13. De Nil LF, Sasisekaran J, Van Lieshout PH, Sandor P. Speech disfluencies in individuals with Tourette syndrome. J Psychosom Res. 2005;58(1):97-102. This study relied on self-reported symptoms - a very interesting methodology. 
14.• Meige H, Feindel E. Tics and their treatment. London: Sidney Appleton; 1907. The ageless classic on tics and TS, unequaled in its breadth of discussion.

15. Barnhill Jr LJ. Improvement in speech dysfluency in Tourette's disorder after tongue piercing. J Am Acad Child Adolesc Psychiatry. 2007;46(1):3-4.

16. Burd L, Freeman RD, Klug MG, Kerbeshian J. Tourette syndrome and learning disabilities. BMC Pediatr. 2005;5:34.

17. Pauls DL, Leckman JF, Cohen DJ. Familial relationship between Gilles de la Tourette's syndrome, attention deficit disorder, learning disabilities, speech disorders, and stuttering. J Am Acad Child Adolesc Psychiatry. 1993;32(5):1044-50.

18. Van Borsel J, Vanryckeghem M. Dysfluency and phonic tics in Tourette syndrome: a case report. J Commun Disord. 2000;33(3): 227-39.

19. Burd L, Kerbeshian J, Cook J, Bornhoeft DM, Fisher W. Tourette disorder in North Dakota. Neurosci Biobehav Rev. 1988;12(3-4): 223-8.

20. Ludlow CL, Polinsky RJ, Caine ED, Bassich CJ, Ebert MH. Language and speech abnormalities in Tourette syndrome. Adv Neurol. 1982;35:351-61.

21. Fisher W, Kerbeshian J, Burd L. A treatable language disorder: pharmacological treatment of pervasive developmental disorder. J Dev Behav Pediatr. 1986;7(2):73-6.

22. Trimble MR, Whurr R, Brookes G, Robertson MM. Vocal tics in Gilles de la Tourette syndrome treated with botulinum toxin injections. Mov Disord. 1998;3(3):617-9.

23. Burd L, Kerbeshian J, Fisher W, Gascon G. Anticonvulsant medications: an iatrogenic cause of tic disorders. Can J Psychiatry. 1986;31(5):419-23.

24. Burd L, Kerbeshian J, Fisher W. Does the use of phenobarbital as an anticonvulsant permanently exacerbate hyperactivity? Can J Psychiatry. 1987;32(1):10-3.

25. Kushner H. A cursing brain? The histories of Tourette syndrome. Cambridge, MA: Harvard University Press; 1999.

26. Freeman RD, Zinner SH, Muller-Vahl KR, Fast DK, Burd LJ, Kano $\mathrm{Y}$, et al. Coprophenomena in Tourette syndrome. Dev Med Child Neurol. 2009;51(3):218-27. A unique study of coprophenomena using TIC sites for data collection; an international perspective on coprophenomena.
27. Shapiro AK. Gilles de la Tourette syndrome. New York: Raven Press; 1988.

28. Jankovic J, Fahn S. The phenomenology of tics. Mov Disord. 1986;1(1):17-26.

29. Van Lancker D, Cummings JL. Expletives: neurolinguistic and neurobehavioral perspectives on swearing. Brain Res Rev. 1999;31(1):83-104.

30. Lang AE, Consky E, Sandor P. "Signing tics": insights into the pathophysiology of symptoms in Tourette's syndrome. Ann Neurol. 1993;33(2):212-5.

31. Comings DE, Comings BG, Knell E. Hypothesis: Homozygosity in Tourette syndrome. Am J Med Genet. 1989;34(3):413-21.

32. Rickards H. Signing coprolalia and attempts to disguise in a man with prelingual deafness [letter]. Mov Disord. 2001;16(4):790-1.

33. Morris HR. Sign language tics in a prelingually deaf man. Mov Disord. 2000;15(2):318-20.

34. Dalsgaard S, Damm D, Thomsen PH. Gilles de la Tourette syndrome in a child with congenital deafness. Eur Child Adolesc Psychiatry. 2001;10:256-9.

35. El-Assra A. A case of Gilles de la Tourette's syndrome in Saudi Arabia. Br J Psychiatry. 1987;151:397-8.

36. Burd L, Kauffman DW, Kerbeshian J. Tourette syndrome and learning disabilities. J Learn Disabil. 1992;25(9):598-604.

37. Hagin RA, Kugler J. School problems associated with Tourette's Syndrome. In: Friedhoff AJ, Chase TN, editors. Tourette's syndrome and tic disorders: clinical understanding and treatment. New York: Wiley; 1982. p. 323-33.

38. Burd L, Kerbeshian J, Wikenheiser M, Fisher W. A prevalence study of Gilles de la Tourette syndrome in North Dakota schoolage children. J Am Acad Child Adolesc Psychiatry. 1986;25(4): 552-3.

$39 . \bullet$ Dutta N, Cavanna AE. The effectiveness of habit reversal therapy in the treatment of Tourette syndrome and other chronic tic disorders: a systematic review. Funct Neurol. 2013;28(1):7-12. A useful review of a behavioral treatment for tics which appears to have effectiveness equal to many medications.

40. Scahill L, Woods DW, Himle MB, Peterson AL, Wilhelm S, Piacentini JC, et al. Current controversies on the role of behavior therapy in Tourette syndrome. Mov Disord. 2013;28(9): 1179-83. 